\title{
Chapter 19 \\ Radio Frequency Propagation Close to the Human Ear and Accurate Ear Canal Models
}

\author{
Louis Chen, Gerry Eaton, Sergey Makarov, and Gregory Noetscher
}

\subsection{Introduction}

When contemplating on- or near-body wireless networks, many factors contribute to creation of an optimal communications link. Transmission signal interface with the human body in the near-field can contribute to significant antenna mismatch and disruption of the transfer of power between the transmitter and receiver(s). For this reason, realistic and anatomically correct human body models are required when conducting simulations aimed at optimizing a transceiver antenna and characterizing the corresponding link budget. This chapter provides detail on the construction of a CAD-based computational human phantom with highly detail outer ear and ear canal structures and compares the simulated power transmission coefficient to models that do not possess this level of anatomic detail.

\footnotetext{
L. Chen · G. Eaton

Bose Corporation, Framingham, MA, USA

S. Makarov

Massachusetts General Hospital, Boston, MA, USA

Worcester Polytechnic Institute, Worcester, MA, USA

G. Noetscher $(\square)$

Worcester Polytechnic Institute, Worcester, MA, USA

e-mail: gregn@nevaem.com
} 


\subsection{Model Construction}

\subsubsection{Base Model}

Numerous full-body computational phantoms are in use today [1], each with a corresponding resolution and level of anatomical precision; each user must decide which model is most appropriate for the simulation methodology and end application. A non-homogenous, multi-structure model, known as the NELLY model, was the basis for the modifications described below. The NELLY model is CST's implementation of the Visible Human Project Female (VHP-Female) model shown in Fig. 19.1 [2]. This model was constructed by hand segmenting cryosection images provided as part of the National Library of Medicine's multi-modal Visible Human Project (VHP) executed in the 1990s and utilized by thousands of users worldwide for a number of eclectic purposes since its completion. The VHP-Female model is composed of approximately 270 individual components, all manifold surfaces that have been registered via a co-registration process. This model was specifically created to enable cross-platform compatibility from an open source data set. While triangular surface meshes fitting the finite element method are the primary means of construction, these meshes may be voxelized to also allow for time domain based methods of simulation.

While this model is perfectly acceptable for a number of simulation problems, including calculation of specific absorption rate (SAR) [3, 4] and antenna development [5, 6], its anatomical accuracy was lacking for specific applications involving transmission in or around the ear; this can be seen in Fig. 19.2 where fine features around the ear have been removed. The original model was optimized for fast runtimes on applications that required this level of detail. For this reason, the source material was revisited and further segmented to augment the existing model with accurate ear and ear canal features.

\subsubsection{Accurate Ear Canal Segmentation}

Since the VHP cryosection data was used in the creation of the initial model, this data was examined again using the same workflow that enabled creation of the VHP-Female model. This consisted of reviewing 450 full color images, each with a pixel resolution of $0.33 \mathrm{~mm} \times 0.33 \mathrm{~mm}$. Each image was a representation of an axial slice of the original patient, depicting all internal organs and structures. Identification of the ear canal was particularly easy in this format and can be seen clearly in Fig. 19.3, which shows a number of axial slices progressing along the vertical $(z)$ axis.

The VHP model (and consequently, the NELLY model) is akin to the layers of an onion; the outermost skin layer completely surrounds a layer of fat, which completely surrounds a layer of muscle (or "average body") tissue. Contained within this 


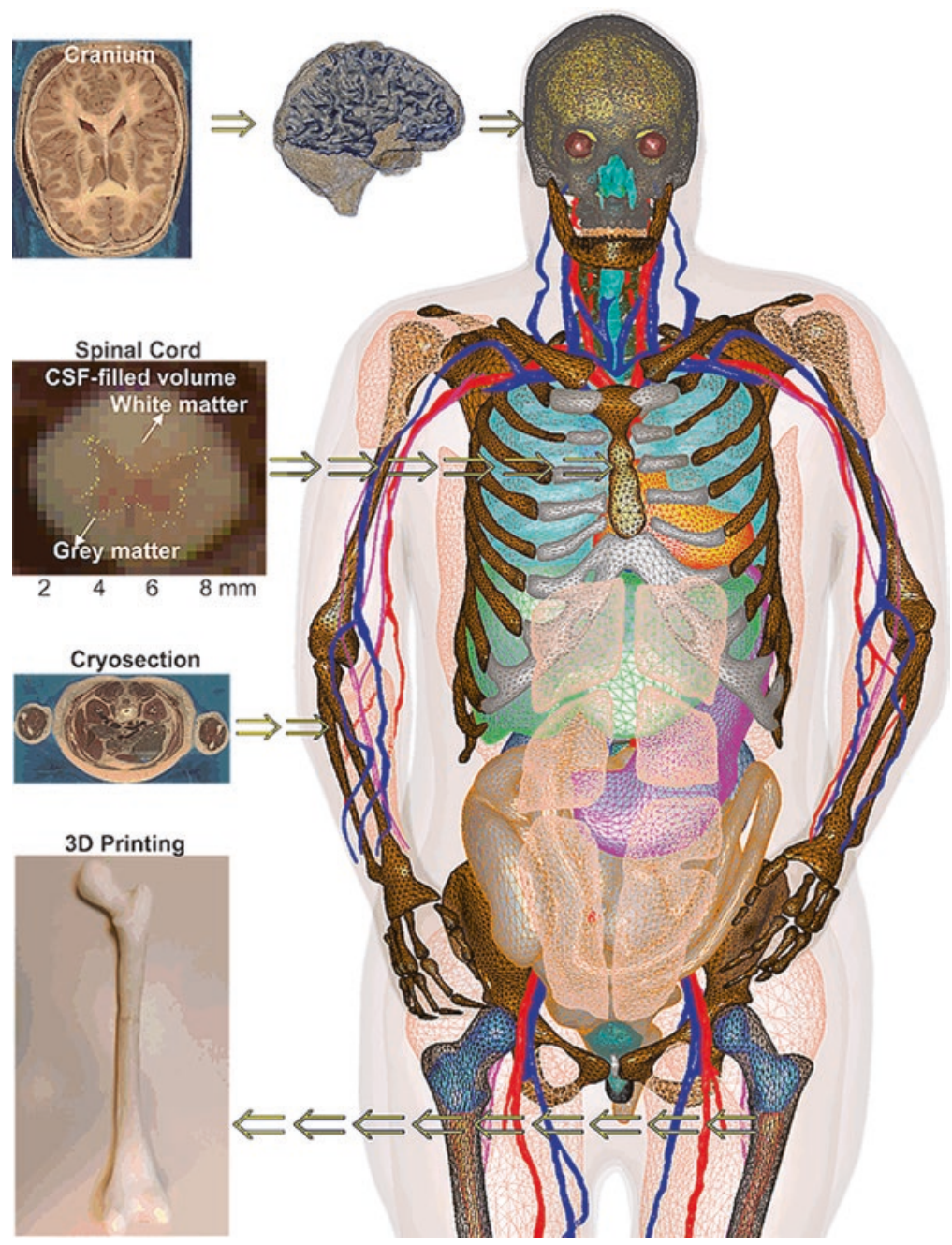

Fig. 19.1 The Visible Human Female model, used as the basis for the NELLY model implemented in CST

muscle layer are all subsequent internal organs and tissues. In order to create a realistic ear and ear canal, the three outermost layers (skin, fat, and average body) required modification to enable integration with the refined features. As shown in Fig. 19.3, the thicknesses between the various layers (skin to fat and fat to muscle) were not homogeneous. This makes sense from an intuitive perspective given that different areas of the body have different material content. Given this fact, the thickness of the skin was tapered from $1 \mathrm{~mm}$ around the subject's scalp to $0.3 \mathrm{~mm}$ within the outer ear. Likewise, the thickness of the fat layer was tapered from $2 \mathrm{~mm}$ to $0.3 \mathrm{~mm}$. 
Fig. 19.2 The original skin shell of the NELLY model, defeatured and optimized for fast simulations
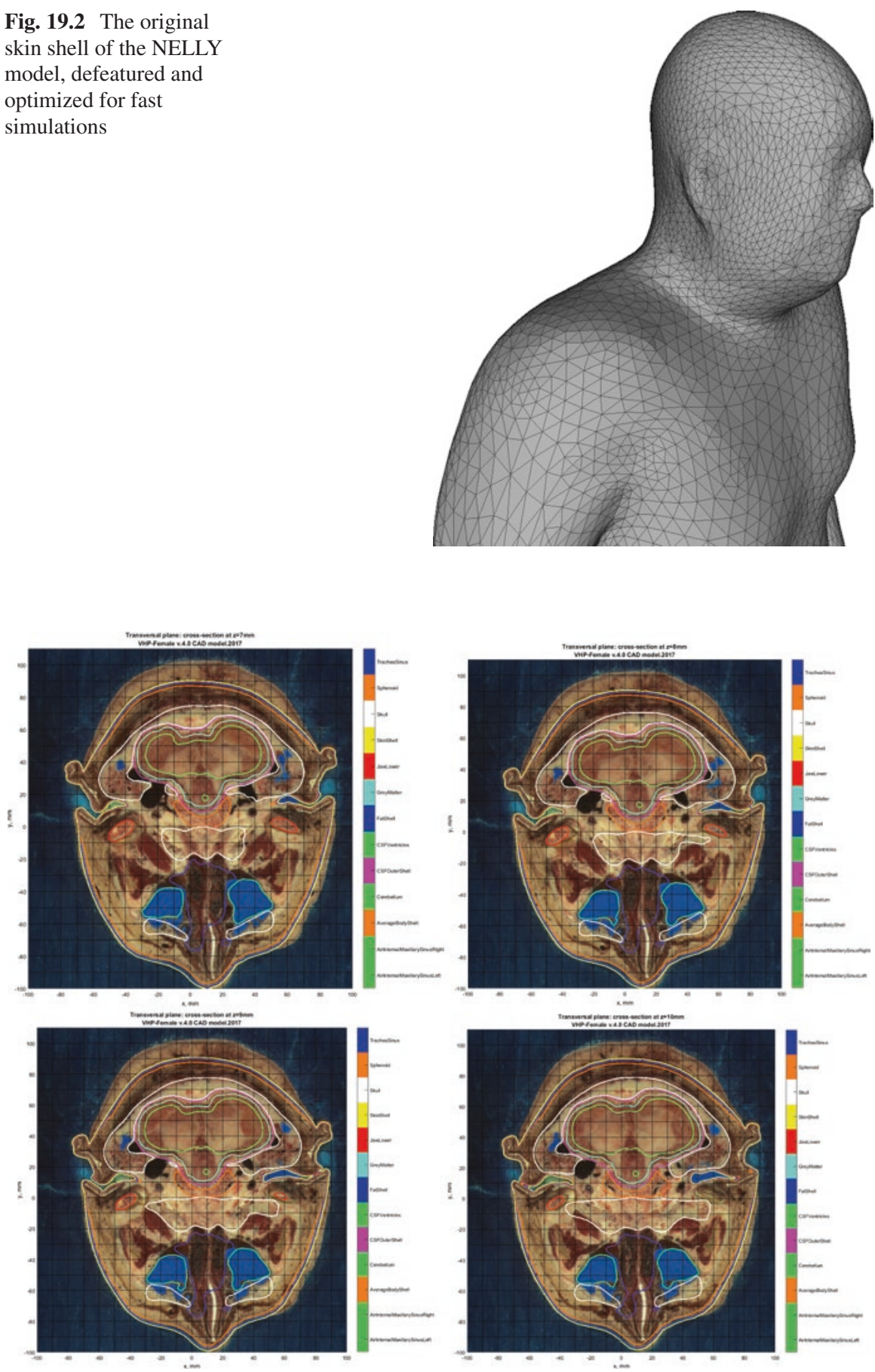

Fig. 19.3 Top row: Segmented cryosection images at $z=7 \mathrm{~mm}$ (left) and $z=8 \mathrm{~mm}$ (right). Bottom row: Segmented cryosection images at $z=9 \mathrm{~mm}$ (left) and $z=10 \mathrm{~mm}$ (right). Layers of the skin, fat and average body container are show in yellow, blue and orange, respectively, with refined outer ear and ear canal structures 


\subsubsection{Levels of Segmentation}

During the segmentation process, the left and right ears were segmented using several levels of refinement. The first segmentation level was highly refined and allowed for the construction of a highly accurate model with over $10 \mathrm{k}$ triangles per ear, shown in Fig. 19.4. Each ear canal was approximately $30 \mathrm{~mm}$ long, stretching into the head and enabling the modeling of this air-filled cavity to assess its impact on antennas placed in or near to the ear.

Following this time-consuming operation, the highly accurate models were decimated via Quadric Edge Decimation to produce left and right components with
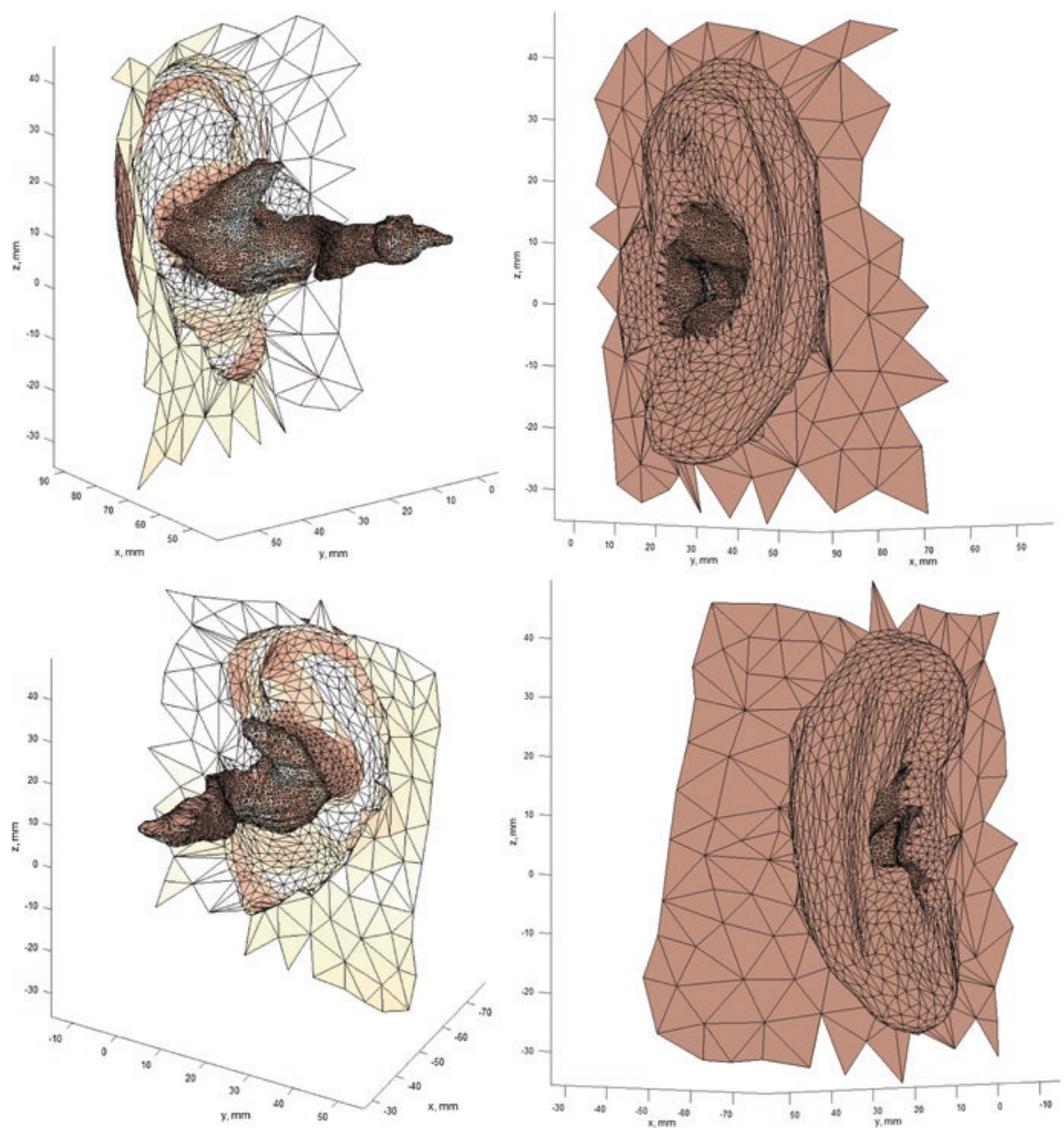

Fig. 19.4 Top row: Highly refined right outer ear (left) and ear canal (right). Bottom row: Highly refined right outer ear (left) and ear canal (right). Not the very high triangle count within the ear canal and stretching approximately $30 \mathrm{~mm}$ into the head 
several hundred triangles each. This decimation was accomplished to enable very fast simulation times when a highly refined model was not necessary. These reduced models are shown in Fig. 19.5.

\subsubsection{Integration of Ear and Ear Canal}

After segmentation and mesh construction of the outer ear and ear canal were completed, these components were stitched together with the skin, fat, and average body shells using the mesh manipulation tool meshmixer. This freeware enables advanced mesh processing and allows for straightforward combination of multiple mesh structures. This operation was validated by overlaying the resulting meshes on top of the cryosection images, as shown in Fig. 19.3. In this way, any user of this product can independently verify that the required accuracy is present in the model.

\subsubsection{Simulation Setup}

The augmented model was read into CST Microwave Studio as a series of stereolithographic model files and complemented with the standard set of human body material properties inherent in the software. A simple simulation designed to establish a wireless link between two antennas located on the body was set up:
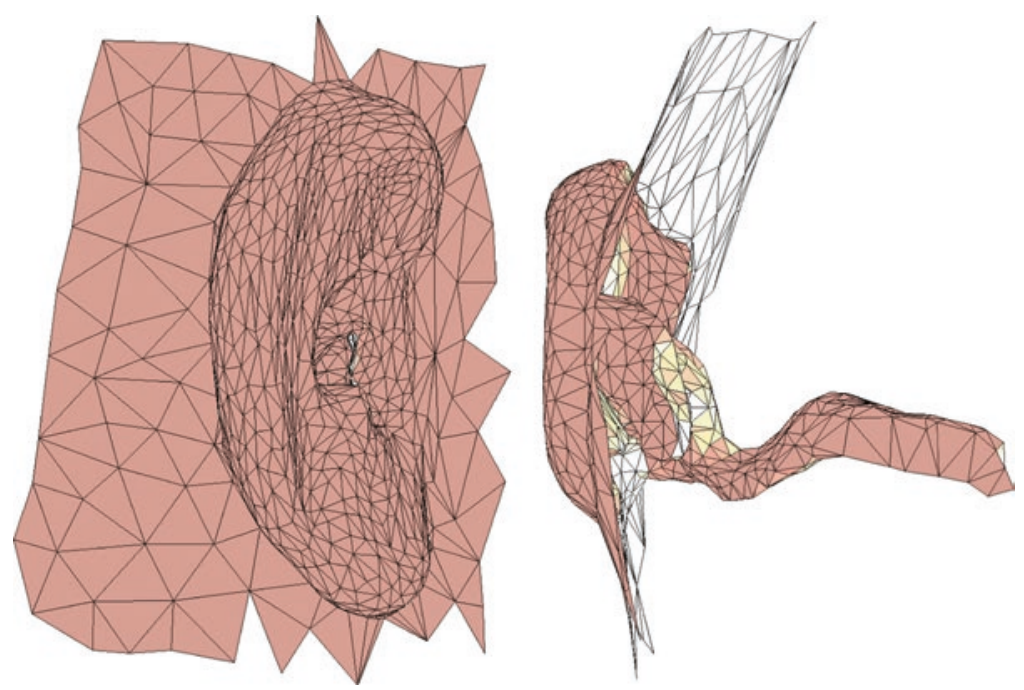

Fig. 19.5 Decimated mesh of the right ear. The number of triangles is drastically reduced from the examples shown in Fig. 19.4 
- Antenna 1 was constructed as a Planar Inverted F Antenna (PIFA). This antenna was located in the right ear and enclosed in a plastic container. This antenna was mirrored in the left ear to obtain transmission coefficient information.

- Antenna 2 was also a PIFA, modeled after a popular cellular phone antenna design. This antenna was placed at the left hip to simulate carrying a phone at this body location.

Table 19.1 below describes the CST simulation settings used for this work.

\subsection{Results}

As outlined in Table 19.1, both the GPU memory usage and the total simulation time were larger when using the augmented model. This was to be expected since a higher global mesh density of six cells per wavelength near the model was used on the augmented model versus four cells per wavelength on the original NELLY. This step was taken due to hardware resource limitations.

The results of the simulations with both models are presented in Table 19.2. It is clear that antenna reflection coefficient is relatively independent of model construction due to similar loading. However, the transmission between the left and right ear is significantly different when incorporating true ear geometry into the model. The transmission from the ear to the pocket is also considerably different with the newly augmented model very closely matching what was experimentally measured.

\subsection{Discussion}

Based on the results shown in Table 19.2, it is clear that including the true anatomic structure into the model will substantially improve the accuracy of the simulation. While this model does require more time and memory to properly simulate, the results are much closer to experiment than those obtained with the previous model.

Table 19.1 Simulation parameters used for the models under discussion

\begin{tabular}{l|l|l}
\hline & Voxel Nelly & Bose-Neva VHP \\
\hline Mesh cells & $896,691,180$ & $566,555,542$ \\
\hline Solver & TLM & TLM \\
\hline GPU & 4 & 4 \\
\hline Mesh cell reduction & $94.3 \%$ & $90.4 \%$ \\
\hline GPU memory usage & $6.7 \mathrm{~GB}$ & $11.3 \mathrm{~GB}$ \\
\hline Total simulation time & $39 \mathrm{~h} 3 \min 22 \mathrm{~s}$ & $49 \mathrm{~h} 13 \min 17 \mathrm{~s}$ \\
\hline
\end{tabular}


Table 19.2 Comparison of simulated results between both models and with experimental measurements

\begin{tabular}{|c|c|c|c|}
\hline \multicolumn{4}{|c|}{ Model comparisons with experiment in $\mathrm{dB}$} \\
\hline & Voxel Nelly & Bose-Neva VHP & Measurement \\
\hline Antenna (in ear) reflection coefficient & -22.4 & -21.3 & - \\
\hline S21 (ear-to-pocket) & -99 & -89 & -88 \\
\hline S21 (ear-to-ear) & -72 & -84 & - \\
\hline
\end{tabular}

One additional source of difference is likely the fact that the surfaces of the Voxel NELLY model are not smooth. Due to its voxel nature, any curved surface on this model is actually a staircase approximation of the true smooth geometry. This may likely play a part in the transmission coefficient calculation and could add further justification for use of a triangular surface based mesh.

\subsection{Conclusions}

This work describes the construction of a finite element compatible model with highly refined outer ear and ear canal structures. This model was created to address the specific need of simulating advanced wireless wearable technologies and to efficiently design in virtual space wearable antennas for on-body propagation. Simulation results were shown to closely match experimental measurements.

Future improvements may consist of augmenting this model with internal ear cartilage structures and assessing the impact of ear morphology on antenna performance. This latter item could include examining the size and spacing of the ear relative to the scalp of the model.

\section{References}

1. Makarov, S. N., et al. (2017). Virtual human models for electromagnetic studies and their applications. IEEE Reviews in Biomedical Engineering, 10, 95-121.

2. Tankaria, H., et al. (2016). VHP-female full-body human CAD model for cross-platform FEM simulations - Recent development and validations. In 2016 38th Annual International Conference of the IEEE Engineering in Medicine and Biology Society (pp. 2232-2235). Orlando, FL.

3. Kozlov, M., Tankaria, H., Noetscher, G., \& Makarov, S. (2017). Comparative analysis of different versions of a human model located inside a 1.5T MRI whole body RF coil. In 39th Annual International Conference of the IEEE Engineering in Medicine and Biology Society (EMBC 2017). Jeju Island, Korea.

4. Kozlov, M., Bazin, P. -L., Möller, H. E., \& Weiskopf, N. (2016). Influence of cerebrospinal fluid on specific absorption rate generated by $300 \mathrm{MHz}$ MRI transmit array. In 2016 10th European conference on antennas and propagation (EuCAP) (pp. 1-5). Davos. 
5. Noetscher, G. M., et al. (2017). Virtual humans for antenna/implant modeling. In 2017 IEEE international symposium on antennas and propagation \& USNC/URSI National Radio Science Meeting (pp. 223-224). San Diego: CA.

6. Venkatasubramanian, A., \& Blair, C. (2015). Modeling and design of antennas for implantable telemetry applications. In 2015 IEEE 16th annual wireless and microwave technology conference (WAMICON) (pp. 1-5). Cocoa Beach, FL. https://doi.org/10.1109/ WAMICON.2015.7120355.

Open Access This chapter is licensed under the terms of the Creative Commons Attribution 4.0 International License (http://creativecommons.org/licenses/by/4.0/), which permits use, sharing, adaptation, distribution and reproduction in any medium or format, as long as you give appropriate credit to the original author(s) and the source, provide a link to the Creative Commons license and indicate if changes were made.

The images or other third party material in this chapter are included in the chapter's Creative Commons license, unless indicated otherwise in a credit line to the material. If material is not included in the chapter's Creative Commons license and your intended use is not permitted by statutory regulation or exceeds the permitted use, you will need to obtain permission directly from the copyright holder.

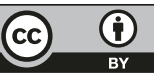

